\title{
Physician Experience With Viewing Digital Radiographs in an Intensive Care Unit Environment
}

\author{
Louis M. Humphrey, Kevin Fitzpatrick, Susan S. Paine, and Carl E. Ravin
}

\begin{abstract}
After several years of continuous operation, the utility of digital viewing stations as assessed by bedside clinicians has been investigated through the distribution of questionnaires to past and present users. The results of the questionnaire have indicated that the bedside physicians prefer using the workstations over handling film. For evaluation of line placements, chest tubes, and pleural effusions, the physicians prefer softcopy display over hardcopy. However, for analysis of air space disease and diagnosis of pneumothorax, images displayed on the workstation were not believed to be as useful as standard hardcopy.

Copyright 1993 by W.B. Saunders Company
\end{abstract}

KEY WORDS: intensive care unit (ICU), picture archiving and communications system (PACS), digital radiography.

QINCE ITS implementation in 1989, a fully $N$ integrated combination of computed digital radiography and picture archiving and communications systems (PACS) for intensive care unit (ICU) radiography has changed the way the effected ICUs use radiology data. Computed digital radiographs (CRs) have provided very consistent and reproducible examinations, markedly improving the ability of both the radiologist and the clinician to assess the effects of treatment between examinations. The addition of a viewing station that allows the clinician to view the images in the ICU itself has further provided significant convenience to the bedside physician.

The system evaluated in this report takes advantage of the integration of computed radiology and PACS technology to provide rapid access to radiology data directly in the ICU. In contrast to some other methods of transmitting information to an $\mathrm{ICU}^{1-4}$ or other location, this system provides a totally digital data stream, from initial processing of the image plates through transmission to the unit and final dis-

From the Department of Radiology and the Department of Surgery, Duke University Medical Center, Durham, NC.

Address reprint requests to Louis $M$. Humphrey, $M A$, Department of Radiology, Duke University Medical Center, $P O$ Box 3808, Erwin Rd, Durham, NC 27710.

Copyright 11993 by W.B. Saunders Company

0897-1889/93/0601-0009\$03.00/0 play. It also requires no manual intervention by personnel in either the ICU or the radiology department to guarantee the transmission of the images. Consequently, the operation has been well received by users in both the ICUs and the radiology department. In both cases, the effort required to provide the clinician with access to digital data has been far less than that required to get the same information to the clinician without digital access. The integration of the systems was so complete that users often had difficulty knowing where digital radiography ended and PACS began. The integrated system is shown in Fig 1 and has been described in greater detail elsewhere. ${ }^{5.6}$ During normal operations, the technologists performing the examinations need only interact with the digital radiography system (Philips Medical Systems, Shelton, CT). Images are automatically routed to the correct location through the PACS based on patient demographic data entered at the digital radiography system. In the ICU, clinicians and other users only interact with their display system to view images for their patients.

The system in use has changed very little since it was first introduced on the two ICUsthe Cardiac Care Unit (CCU, a medical unit) and the Surgical ICU (SICU, a surgical unit). These units were targeted to allow assessment of potentially differing patterns of utilization and acceptance that might occur between medical and surgical units.

This study was designed to assess clinician perceptions of the system and its utility relative to conventional plain film-based ICU operations.

\section{DESCRIPTION OF THE INTENSIVE CARE DISPLAY ENVIRONMENTS}

Each of the ICUs is a 16-bed unit with an average occupancy of between 13 and 15 patients and an average length of stay varying from 3 to 7 days. Both units occasionally have patients who stay on the unit for extended periods ( $>20$ days). Although each unit usually performs the same type of examinations for rounds and immediate examinations (mostly chest), the information is often accessed differently. 


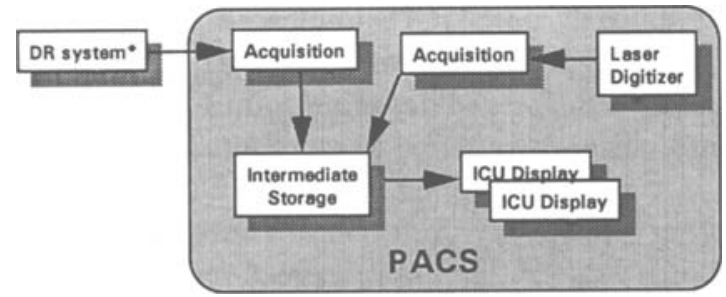

Fig 1. Block diagram of integrated digital radiography/ PACS used for ICU transmissions (The Philips Computed Radiography System [PCR-901]].

As shown in Fig 2, most of the viewing on the SICU occurs during the day, whereas the viewing on the CCU occurs more often at night. In addition, the number of instances in which the images are viewed is generally higher on the SICU than the CCU throughout the day. Nonetheless, both units consider the systems to be essential. Since starting digital access, neither unit has used film copies except on those few occasions when some part of the system was not working. Standard radiographic view boxes that had been used on the SICU have been removed. Daily conferences with CCU physicians, in which the hard copy (display film) of ICU patients are reviewed, have continued to be held in the radiology department.

The display systems are centrally located on each unit. In each case, the display monitors are mounted above a central nursing station. This allows easy access from any location in the unit. The monitors are arranged so that the images are easily viewed by several people at once. This has made the system ideal for rounds as well as normal individual viewing. The systems have been used mostly by ICU physicians, but were also available to other users in the ICUs, such as nurses and physicians' assistants.

\section{MATERIALS AND METHODS}

Details of the system being evaluated have been described in detail elsewhere..$^{5,6}$ The integrated system consists of three essential components: a computed radiography system (the PCR-901, Philips, Shelton, CT), a PACSstyle acquisition and storage system, and an ICU display system. The computed radiography system was used, along with its components, for image acquisition and processing. The acquired images were then transmitted to the PACS through two interface components, one on the digital radiography side of the interface and another on the PACS side. The PACS included a central storage component for archival storage and routing. The images were automatically routed to the display system on the appropriate ICU. Images transmitted to the PACS from the digital radiography system were transmitted over a DR-11W interface. Internal communications within the PACS use fiber optic connections with tightly coupled, proprietary transmission protocols that are optimized for speed. Transmissions to the ICUs used standard Transmission Control Protocol/ Internet Protocol (TCP/IP) on Ethernet. The images transmitted to the ICUs were spacially and contrast reduced from an initial $2,000 \times 2,000 \times 10$-bit matrix (as output from the digital radiography system) to a final 1,000 pixels $\times$ 1,000 pixels $\times 8$-bit matrix. Examinations for patients on each unit were automatically transmitted to the viewing stations as soon as the images were processed. The transmitted images were stored locally for easy viewing on two 1,000 pixel $\times 1,000$-pixel monitors.

To assess the utility of the systems in the ICUs, question-
Fig 2. Average number of uses of the digital viewing station as a function of time during the day

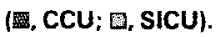

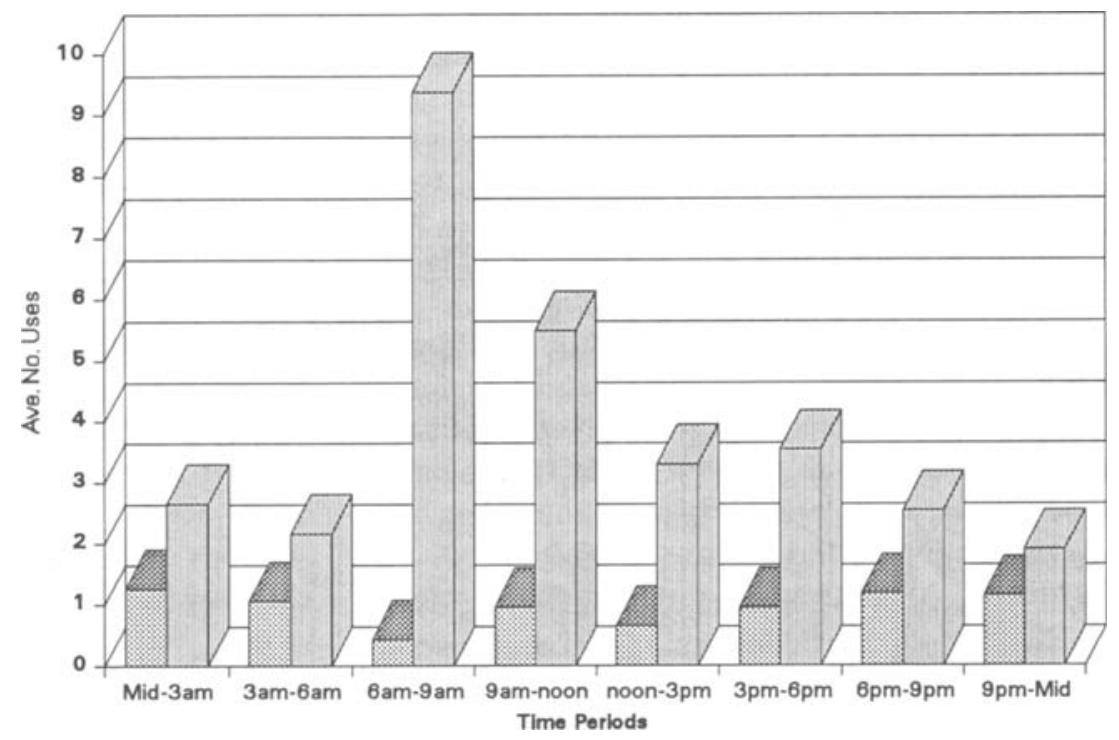


naires were sent to 99 medical and surgical residents and faculty who were known to have rotated through the units that were equipped with the digital systems. The majority of the clinicians to whom the questionnaire was sent used the digital viewing stations for image display for a total of 1 to 2 months while on one of the two equipped units as compared with 10 to 11 months spent on units without digital viewing capabilities. Consequently, over a period of 1 year, the respondents tended to spend the majority of their time on units without digital viewing stations. Each questionnaire contained five parts to assess perceived differences between softcopy and hardcopy viewing for different types of disease processes, ability to use controls, and timeliness of transmissions as a function of user type and ICU served. The questions and structure of the questionnaire were reviewed by members of a business school (The Fuqua School of Business, Duke University, Durham, NC). The questionnaire (reproduced in the appendix) was brief-limited to two pages to encourage the return of as many as possible.

Once the questionnaires were returned, the data were compiled and analyzed using standard statistical methods. In applicable areas, a multivariate analysis was applied

\section{RESULTS}

Of the 99 questionnaires sent to ICU clinicians, 47 were returned. Approximately $70 \%$ of the returned questionnaires were from residents, $18 \%$ were from faculty and fellows, and in $12 \%$ the status of the respondent could not be determined.

One of the major sections of the questionnaire dealt with the comfort and ability of the respondents to use the digital viewing stations for all disease processes and patient treatment. Using hardcopy as the standard, the respondents were asked to indicate their preferences for hardcopy over softcopy for several disease processes and treatments that were normally assessed or monitored by portable chest radiography. The disease processes and treatments included were line placements, chest tube placements, pneumothorax, airspace disease, and pleural effusions.

Responses for these disease processes are displayed graphically in Fig 3. There was a general preference for hardcopy when considering lung-related diseases, although those preferences were more pronounced for the CCU than the SICU. For evaluation of chest tubes, line placements, and pleural effusions, there was an overwhelming preference was for softcopy over hardcopy. A multivariate analysis of the vari- ance using both ICUs indicated that preferences fell into two clusters. Chest tube placements, line placements, and effusions fell into one cluster-softcopy preference, whereas air space disease and pneumothorax fell into the other-hardcopy preference. There was no significant difference in preference for softcopy in evaluation of chest tube placements, line placements or pleural effusions. Similarly, there is no significant difference in preference between air space disease and pneumothorax. However, the preference for hardcopy over softcopy for air space disease and pneumothorax was significantly different from the preference for softcopy over hardcopy for line placements, effusions, and chest tube placements. For all categories of users-faculty, senior residents, and junior residents-the results were essentially the same. The most noticeable difference in the three groups was in the preferences for hardcopy for diagnosing air space disease. Fellows and faculty preferred softcopy more often than either group of residents $(\mathrm{P}=.27)$. Respondents were asked to indicate a preference for hardcopy over softcopy when looking for five different disease processes and treatments: chest tube placements, pleural effusions, air space disease, line placements, and pneumothorax. These disease processes and treatments can be divided into two general groups: lung-related diseases (air space, effusions, and pneumothorax) and external devices (lines and tubes). There was a definite preference for hardcopy in two categories: air space disease and pneumothorax, for which the mean preferences were $52.2 \%$ and $65.2 \%$, respectively.

Fig 4 shows a graphical breakdown of the five clinical categories for the two ICUs. The preference for hardcopy when looking for pneumothorax and air space disease is easily seen here, although these preferences are more pronounced for the CCU than for the SICU. Additionally, the respondents who work in the CCU show an overall preference for hardcopy over those from the SICU. The respondents from the SICU were $4^{1 / 4}$ times more likely to prefer softcopy over hardcopy for the air space disease category than were those from the CCU.

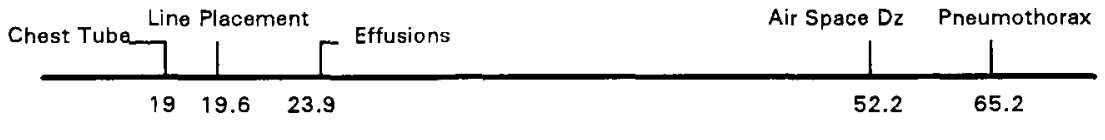

Fig 3. Mean preference for hardcopy over softcopy $(\%)$. 


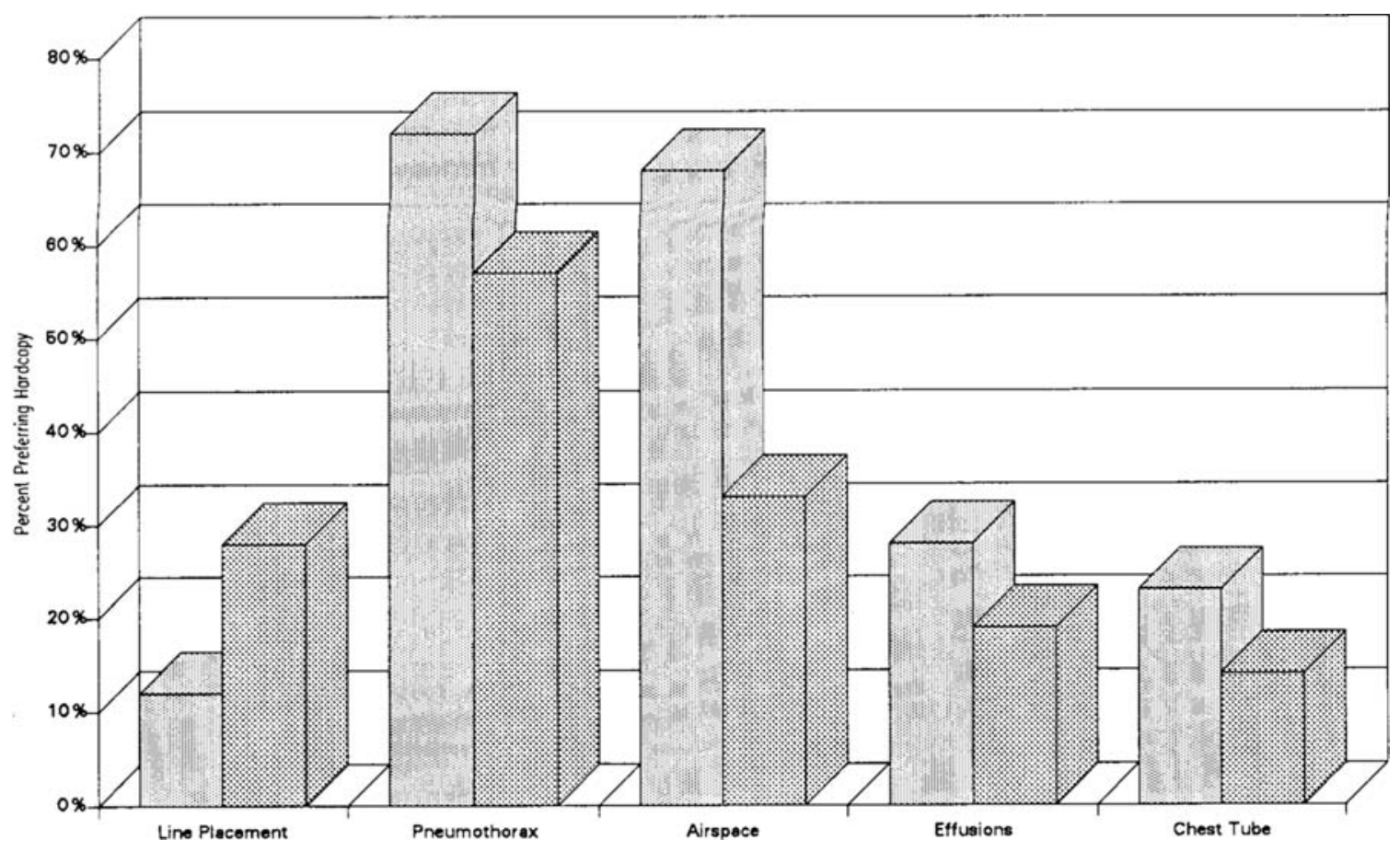

Fig 4. Percentage of respondents from each ICU preferring hardcopy over softcopy for five clinical categories (⿶, CCU, $\square$, SICU).

With regard to the digital viewing station, the respondents seemed satisfied with the controls used for both the general workstation controls and the image adjustment controls (window/ level, orientation, etc). These results are shown in Fig 5 for the respondents as a group.

Before the digital systems were added to the ICUs, both units received film copies of examinations performed on patients on the unit. In addition, whenever the digital system was inoperative, copies of film were sent to the unit. When asked about their perception of arrival times of the hardcopy films versus softcopy images, most of the respondents agreed that softcopy arrived faster most of the time. When asked if the digital system transmitted the images in a timely fashion, most respondents also indicated that the images were available in time to be of use in determining the best course of treatment for the patient. These responses are shown in Fig 6. Again, results for both ICUs have been combined because there were no significant differences between them.

\section{DISCUSSION}

The questionnaires returned by the ICU clinicians show a general preference for digital viewing stations over conventional film and the related delays in receiving radiology information. There was a preference for hardcopy for lung-related diseases. The images transmitted to both ICUs are generated by the Philips 901 digital radiography system. With this system, two images are routinely produced from each exposure. One image is processed to provide a film image close to that routinely produced with plain film. The other image is processed with additional edge-enhancement to make it easier to locate lines and tubes. Both of these images are printed on a single sheet of film for use by the radiologist. However, only the edge-enhanced image was transmitted to the ICUs. No effort was made to modify the enhancement settings to provide optimum softcopy viewing for both lung diseases and external devices. Thus, it is not entirely surprising that hardcopy seemed preferable for analysis of lung disease which is more difficult to assess on edgeenhanced images. The lung-related diseases group also had the widest dispersion of all groups, indicating that it should be possible to configure the image sent to the ICUs such that lung-related diseases would also be easily detected using softcopy without significantly affecting the ability to detect lines and tubes.

Apart from the differences in usage patterns 


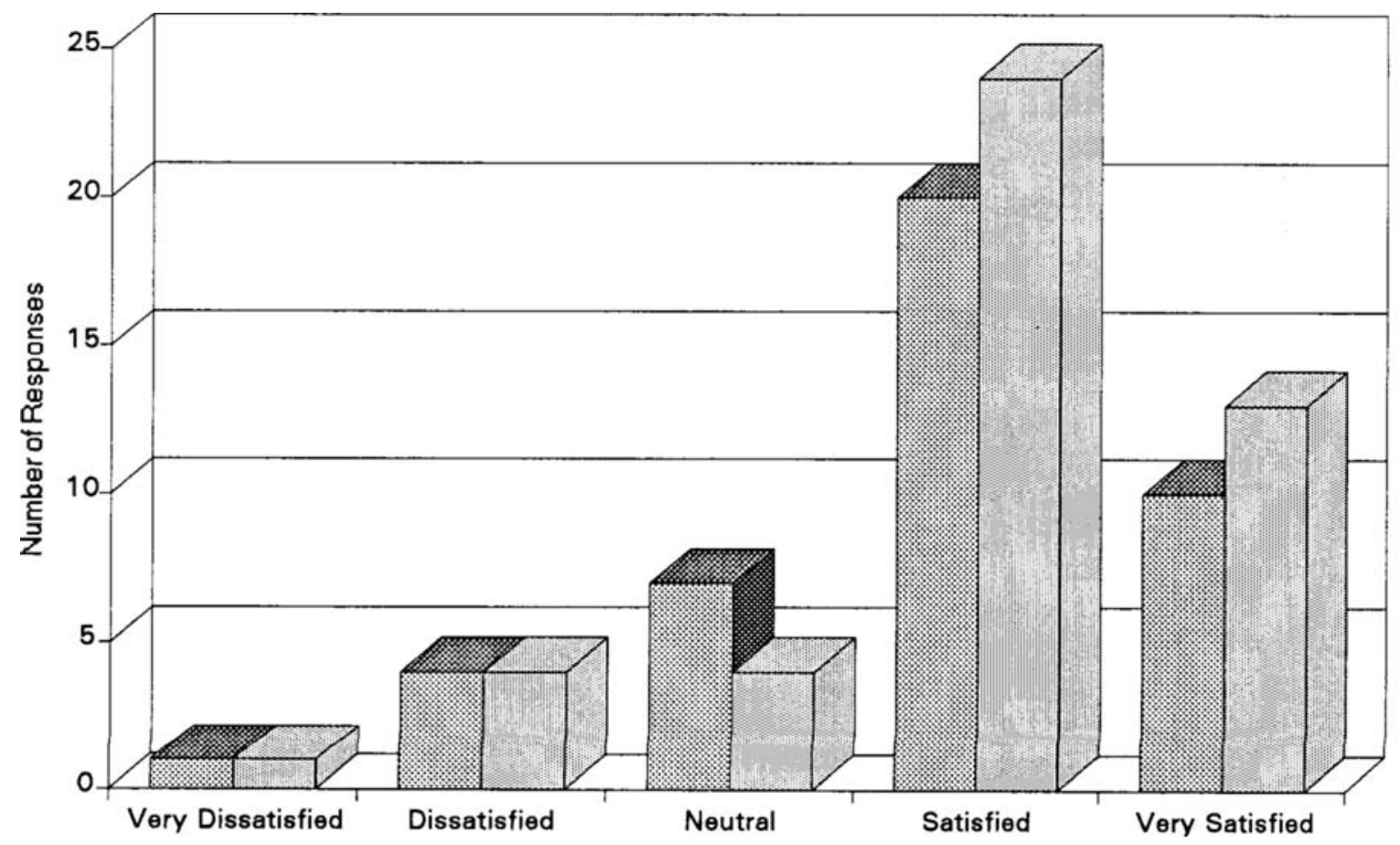

Fig 5. Level of satisfaction of respondents with workstation controls for image manipulation and general operation. (囲, image control; $\square$, station control).

and frequency of use for the two units (Fig 2), most other categories were the same for both the CCU and the SICU. Because the primary focus of $\mathrm{CCU}$ physicians is more often lung- related disease processes than the SICU physicians, it is easy to understand their stronger preference for hardcopy in these cases (Fig 4).

The general ability of the respondents to use

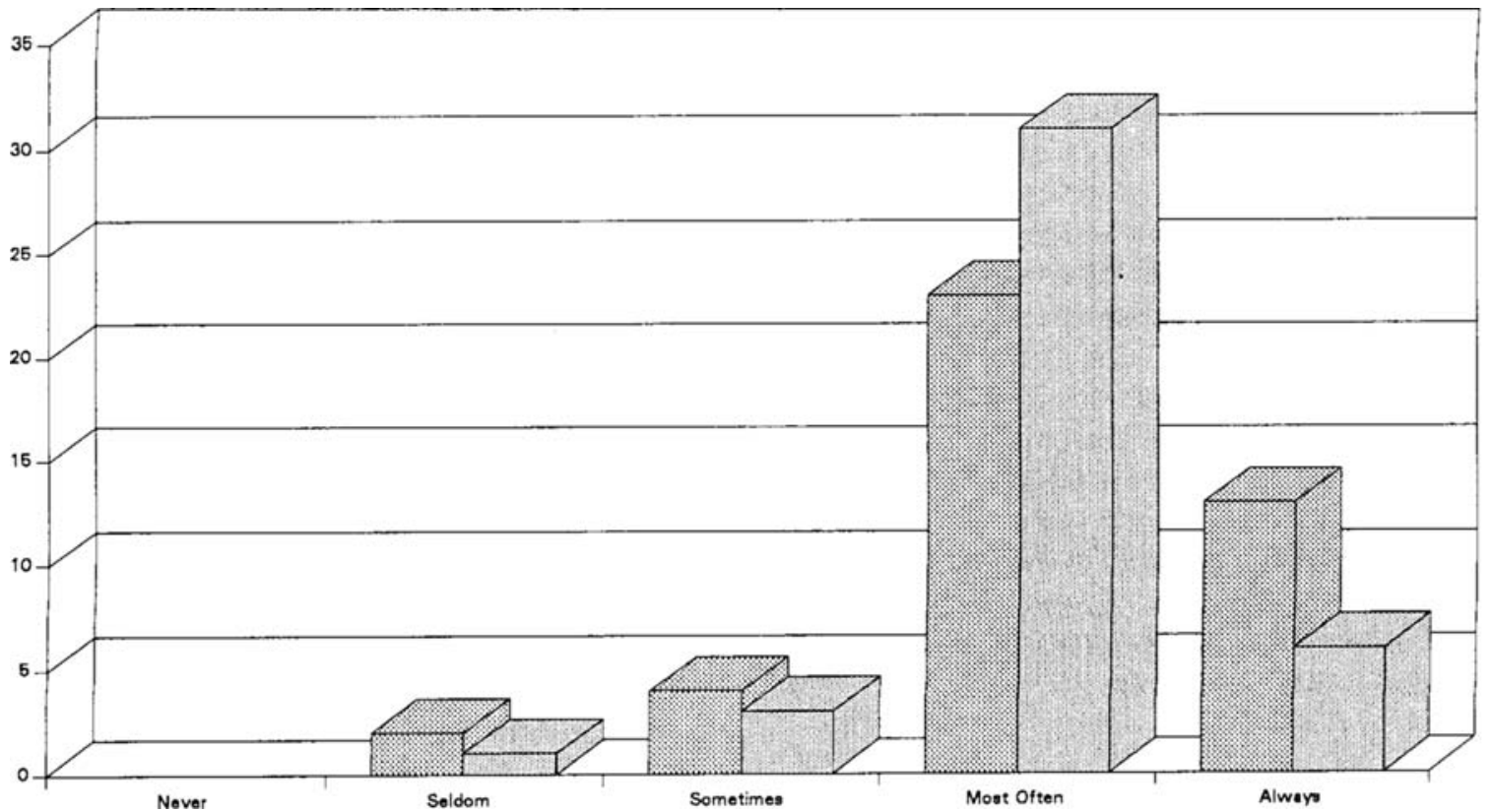

Fig 6. Perceived differences in speed of transmission and availability of softcopy versus hardcopy (閣, fast softcopy; $\square$, fast transmission). 
the workstation effectively was confirmed by the questionnaire results. Simple capabilities needed to view and manipulate images seem to be important. In general, the users indicated satisfaction with the present user interface, even though it has not changed significantly in several years. There was also the desire from most respondents for additional capability, such as a listing of examinations from which choices could be made or selection of a particular body region or examination type for display.

The perceptions of the timeliness of images arriving on the units were as expected. In most cases, the images arrived on the unit within minutes of the time the images were processed. This represented a significant improvement over the previous system, in which copies were made and sent to the units up to several hours later.

\section{DUKE MEDICAL CENTER}

DIGITAL RADIOGRAPHY QUESTIONNAIRE

The primary location for my digital imaging workstation is: SICU $\mathrm{CCU}$

Please indicate your position at Duke (Intern, JAR, SAR, Fellow, Faculty, etc)

Please circle the number that best describes your opinion about the digital imaging workstations.

1. The following questions pertain to availability, accessibility, and ease of use:

$\begin{array}{ccccc}\text { Never } & \text { Seldom } & \text { Sometimes } & \begin{array}{c}\text { Most } \\ \text { Often }\end{array} & \text { Always } \\ 1 & 2 & 3 & 4 & 5 \\ 1 & 2 & 3 & 4 & 5\end{array}$

2. With respect to overall image quality, please state your preferred imaging form for the following clinical indications.

a. Are portable films available at the viewing station in a timely fashion?

Digital Image

Standard Hardcopy

No Difference
a. Line placement
b. Pneumothorax
c. Airspace disease
d. Effusions
e. Chest tube placements

1

1

1

$\begin{array}{ll}2 & 3 \\ 2 & 3 \\ 2 & 3 \\ 2 & 3 \\ 2 & 3\end{array}$

3. Do you prefer working in an ICU WITH or WITHOUT Digital Imaging Capability (Check One). With Digital Images Without Digital Images No Difference

4. Are there any clinical areas other than the ICUs where digital radiography might be useful?

NO _ _ YES (Please specify)

5. Please indicate your overall satisfaction/dissatisfaction with the digital radiography imaging system.

\begin{tabular}{ccccc}
$\begin{array}{c}\text { Very } \\
\text { Dissatisfied }\end{array}$ & Dissatisfied & Neutral & Satisfied & $\begin{array}{c}\text { Very } \\
\text { Satisfied }\end{array}$ \\
1 & 2 & 3 & 4 & 5 \\
1 & 2 & 3 & 4 & 5 \\
1 & 2 & 3 & 4 & 5 \\
\hline
\end{tabular}

To what extent are you satisfied with the radiography workstation?

How would you describe your satisfaction with digital radiography workstations to your colleagues in other hospitals?

To what extent are you satisfied with viewing station controls? 


\section{DUKE MEDICAL CENTER \\ DIGITAL RADIOGRAPHY QUESTIONNAIRE (Cont'd)}

6. How would you describe your interaction with Radiologists when on units using the digital radiography system?

$\begin{array}{cc}\text { Much } & \text { Slightly } \\ \text { Less } & \text { Less }\end{array}$

No

Slightly

Much

Please use the additional space here for other comments.

Thank you for your participation in our assessment of digital radiography.

\section{REFERENCES}

1. Arenson RL, Seshadri SB, Kundel HL, et al: Clinical evaluation of a medical image management system for chest images. AJR Am J Roentgenol 150:55-59, 1988

2. Franken EA Jr, Smith WL, Berbaum KS, et al: Compatison of a PACS workstation with conventional film for interpretation of neonatal examinations: A paired comparison study. Pediatr Radiol 21:336-340, 1991

3. DeSimone D, Kundel HL, Arenson RL, et al: Effect of a digital imaging network on physician behavior in an intensive care unit. Radiology 169:41-44, 1988
4. Gillespy T, Staab EV, Lawrence E: Electronic imaging in a teaching hospital intensive care unit: Evaluation of the clinical review system. J Digit Imaging 3:124-128, 1990

5. Ravin CE: Initial experience with automatic image transmission to an intensive care unit using picture archiving and communications system technology. J Digit Imaging 3:195-199, 1990

6. Whitt RM, Cohen MD, Appledorn CR: Initial experience with a radiology imaging network to newborn and intensive care units. J Digit Imaging 4:39-42, 1991 